\title{
Atypical cutaneous features in a patient with adult-onset Still's disease
}

\author{
Woo Seong Jeong ${ }^{1}$, Jinseok Kim ${ }^{1}$, Sang Taek $\mathrm{Heo}^{1}$, Jae Wang Kim² ${ }^{2}$, and Jeong Rae Yoo
}

Departments of ${ }^{1}$ Internal Medicine and ${ }^{2}$ Dermatology, Jeju National University School of Medicine, Jeju, Korea

Received: November 19, 2019 Revised : December 3, 2019 Accepted: December 3, 2019

\section{Correspondence to} Jeong Rae Yoo, M.D.

Tel: +82-64-717-2283

Fax: +82-64-717-1131

E-mail: mdyoojr@gmail.com https://orcid.org/0000-00025488-7925
A 77-year-old woman with fever $\left(39 \cdot 5^{\circ} \mathrm{C}\right)$ and skin rash who was previously healthy was admitted, except for hypertension. Physical examination revealed polymorphrous erythematous coalescent figurate edematous papular plaques of 3-week duration over the lower lumbar region (Fig. 1A). Ten days prior, she reported symptoms of myalgia, arthralgia, and sore throat with fever. Following admission, she was examined for fever of unknown origin.

Blood test results were as follows: white blood cell count, $21.8 \times 10^{3} / \mu \mathrm{L}$ (4.0 to 10.0); erythrocyte sedimentation rate, $93 \mathrm{~mm} / \mathrm{hr}$ (o to 20); C-reactive protein, $18.86 \mathrm{mg} / \mathrm{dL}$ (o.00 to 0.30 ); ferritin, $1,675.56 \mathrm{ng} / \mathrm{mL}$ (4.63 to 204.00); aspartate transaminase, $116 \mathrm{IU} / \mathrm{L}(8$ to 38); alanine aminotransferase, 102 IU/L (4 to 44 ); interleukin $6,87.4 \mathrm{pg} /$
$\mathrm{mL}$ ( $\leq 7.0)$. Microbiological and autoimmune assays were unremarkable. On admission day, she underwent a lower abdominal region skin biopsy. On hospital day 6 , the skin rash, high fever, and arthralgia were persistent, and 18F-fluorodeoxyglucose positron emission tomography/computed tomography revealed multiple lymph node enlargement and splenomegaly. Histology results showed lymphohistiocytic infiltration and multiple individual necrotic keratinocytes in the upper epidermis, stromal leukocytoclasia, dermal mucin deposition, and basal vacuolar alteration consistent with pathologic findings of adult-onset Still's disease (AOSD) (Fig. 2). Following AOSD diagnosis, prednisolone $25 \mathrm{mg}$ twice a day was started, and the myalgia, arthralgia, fever, skin rash,
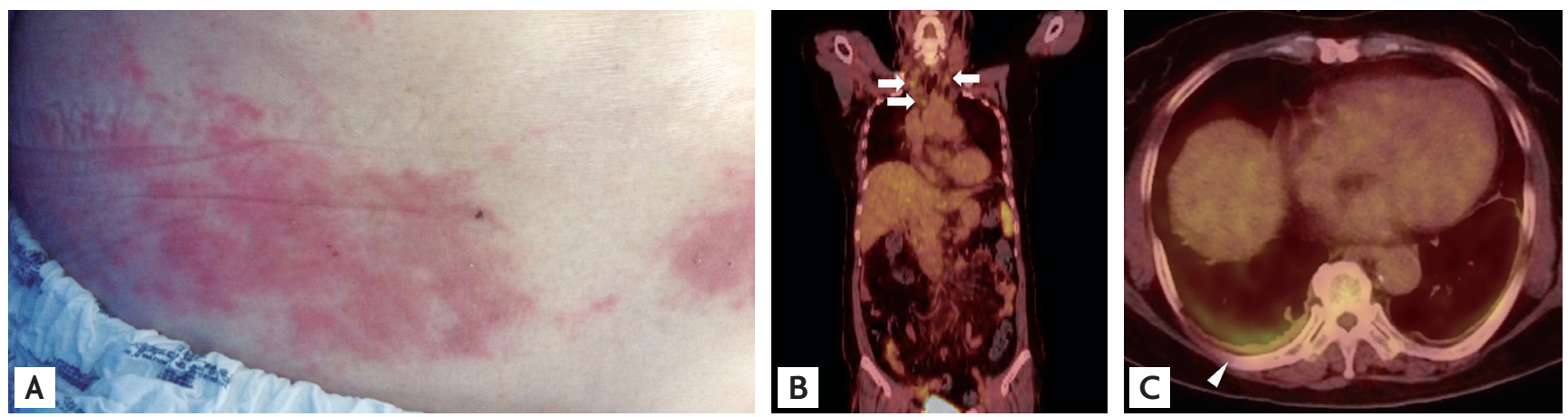

Figure 1. (A) Polymorphous erythematous coalescent figurate edematous papular plaques observed on the lower back at admission. (B) Positron emission tomography-computed tomography findings show multiple lymph node enlargements on the supraclavicular and paratracheal areas (white arrows). (C) A small amount of effusion and fluorodeoxyglucose uptake in the left pleura (white arrowhead). 

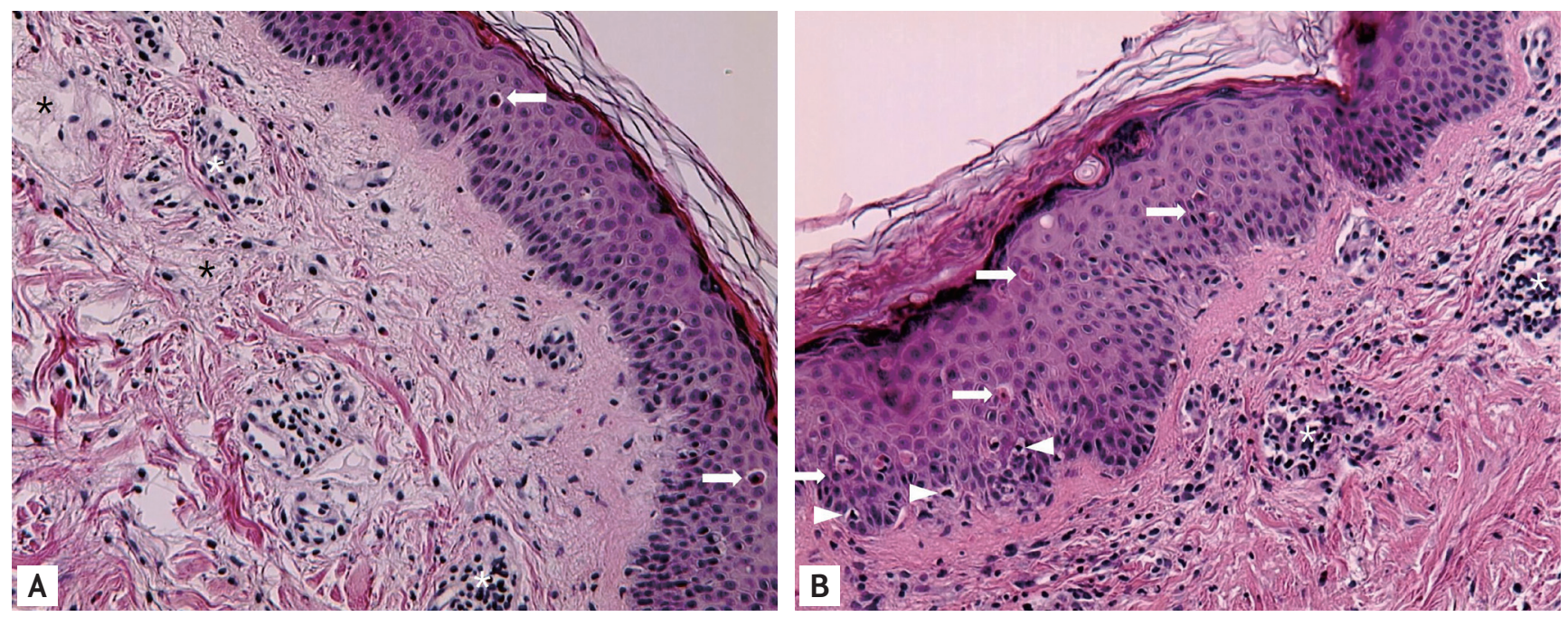

Figure 2. (A) Multiple individual necrotic keratinocytes singly or in aggregates are observed mainly in the upper epidermis (arrows). Mixed cellular perivascular and interstitial infiltrates are composed of neutrophils, lymphocytes, and histiocytes. Stromal leukocytoclasia (nuclear dust), eccrine hidradenitis (white asterisks), and dermal mucin deposition (black asterisks) are observed. (B) Arrowheads indicate basal vacuolar alteration. Arrows indicate multiple epidermal apoptotic keratinocytes $(\mathrm{H} \& \mathrm{E}, \times 100)$.

and blood test results improved (Fig. 1B). The skin rash did not appear with typical distinctive evanescent salmon-pink erythema. However, the patient met AOSD diagnostic criteria, namely, high fever, leukocytosis, sore throat, multiple lymph node enlargements, hepatic dysfunction, splenomegaly, and negative rheumatoid factor and antinuclear antibody findings. Additionally, she had a serositis on the pleura (Fig. 1C).

AOSD is a systemic inflammatory disorder of unknown etiology. Diagnosis was difficult because of var- ious nonspecific signs and symptoms and because no disease-specific diagnostic tests are available. Skin biopsy is recommended for patients with an atypical cutaneous presentation.

Written informed consent was obtained from patient.

\section{Conflict of interest}

No potential conflict of interest relevant to this article was reported. 